Portland State University

PDXScholar

\title{
Automated Crystal Phase and Orientation Mapping of Nanocrystals in a Transmission Electron Microscope
}

\author{
Peter Moeck \\ Portland State University, pmoeck@pdx.edu \\ Sergei Rouvimov \\ Portland State University \\ Edgar F. Rauch \\ SIMAP/GPM2 Laboratory \\ Stavros Nicolopoulos \\ NanoMEGAS SPRL
}

Follow this and additional works at: https://pdxscholar.library.pdx.edu/phy_fac

Part of the Nanoscience and Nanotechnology Commons, and the Physics Commons Let us know how access to this document benefits you.

\section{Citation Details}

Moeck, P., Rouvimov, S., Rauch, E. F., \& Nicolopoulos, S. (2009, September). Automated crystal phase and orientation mapping of nanocrystals in a transmission electron microscope. In Frontiers of Characterization and Metrology for Nanaoelectronics: 2009 (Vol. 1173, No. 1, pp. 304-308). AIP Publishing.

This Conference Proceeding is brought to you for free and open access. It has been accepted for inclusion in Physics Faculty Publications and Presentations by an authorized administrator of PDXScholar. Please contact us if we can make this document more accessible: pdxscholar@pdx.edu. 


\title{
Automated crystal phase and orientation mapping of nanocrystals in a transmission electron microscope
}

\author{
Peter Moeck ${ }^{1}$, Sergei Rouvimov ${ }^{1}$, Edgar F. Rauch ${ }^{2}$, and Stavros Nicolopoulos ${ }^{3}$ \\ ${ }^{1}$ Nano-Crystallography Group, Department of Physics, Portland State University, Portland, OR 97207-0751, U.S.A. \\ \& Oregon Nanoscience and Microtechnologies Institute, www.onami.us \\ ${ }^{2}$ SIMAP/GPM2 laboratory, CNRS-Grenoble INP, BP 46101 rue de la Physique, 38402 Saint Martin d'Hères, \\ France \\ ${ }^{3}$ NanoMEGAS SPRL, Boulevard Edmond Machterns No 79, Saint Jean Molenbeek, Brussels, B-1080, Belgium, \\ nanomegas.com
}

\begin{abstract}
An automated technique for the mapping of nanocrystal phases and orientations in a transmission electron microscope (TEM) is described. It is based on the projected reciprocal lattice geometry that is extracted from electron diffraction spot patterns. The required hardware allows for a scanning-precession movement of the primary electron beam on the crystalline sample and can be interfaced to any newer or older TEM. The software that goes with this hardware is flexible in its intake of raw data so that it can also create orientation and phase maps of nanocrystal from high resolution TEM (HRTEM) images. When the nanocrystals possess a structure with a small to medium sized unit cell, e.g. noble metals or minerals that possess the halite structural prototype, an objective-lens aberration corrected microscope needs to be utilize for the recording of the HRTEM images that are to be processed by this software. Experimentally obtained crystal phase and orientation maps are shown for iron oxide and clausthalite nanocrystals. Comprehensive commercial and open-access crystallographic databases that may be used in support of the nanocrystal phase identification process are briefly mentioned.
\end{abstract}

Keywords: structural characterization, nanocrystals, precession electron diffraction, high resolution transmission electron microscopy

PACS: 61.05.jm, 61.46.-w, 61.46.Hk, 61.46.Df, 68.37.Lp, 81.07.Be, 61.68.+n

\section{INTRODUCTION}

The development of novel materials for micro- and nano-electronics requires reliable characterization of crystallite phases, sizes, and mutual orientations in thin polycrystalline films and ensembles of nanocrystals. The electron backscatter diffraction (EBSD) technique (that is also known as orientation imaging microscopy) in a scanning electron microscope (SEM) [1] is often employed for structural characterization of these kinds of materials. EBSD in a SEM is based on backscattered Kikuchi lines. It is therefore highly sensitive to the plastic deformation state of the crystallites as well as to structural damage or contamination of the crystal surfaces.

Electron diffraction in a transmission electron microscope (TEM), on the other hand, delivers a significantly higher spatial resolution as compared to EBSD in a SEM. This is especially true when a field emission gun and very small condenser apertures are utilized. Transmission electron diffraction is also less sensitive to the plastic deformation state and possible surface layers of crystallites. In short, in cases of very small crystallite sizes and film thicknesses, e.g. on the length scale of a few tens of nanometer, the crystal orientation and phase mapping by this technique is intrinsically more reliable than by its EBSD/SEM counterpart. This is especially important for ensembles of nanocrystals which frequently possess a crystallographically distinct surface structure as part of their functionalization [2].

In order to bring the technical advantages of transmission electron diffraction to bear in the routine analysis of nanocrystals in a TEM, the technique needed to be automated. Only then it was able to compete also with the speed and ease of use of the EBSD/SEM technique in the field of crystal phase and orientation mapping of larger crystallites.

In the fall of 2008, such an automated electron diffraction system has been demonstrated for the first time. Heavily deformed $\mathrm{Cu}$ foils with grain sizes on the order of $20 \mathrm{~nm}$ were used as examples [3]. The utilization of the precession electron diffraction mode $[4,5]$ proved crucial to the success of the automated procedure on these samples [3]. Note that the technique is based on template matching of experimentally obtained electron diffraction spot patterns to their precalculated theoretical counterparts. Such template

CP1173, Frontiers of Characterization and Metrology for Nanoelectronics: 2009 
matching has been utilized off line for the crystallographic characterization of precipitates in heavily deformed austenitic stainless steels [6,7] and shown to be a very robust procedure. Precession of the primary electron beam around the microscope's optical axis during the recording of electron diffraction spot patterns results in more reflections with intensities that are closer to the predictions of the kinematical theory $[4,5]$. Its utility for the automated nanocrystal phase and orientation mapping is, therefore, quite obvious.

The Brussels/Belgium based NanoMEGAS* company offers an automated turn-key solution to precession electron diffraction based nanocrystal phase and orientation mapping. This paper describes this company's system briefly and illustrates the application of the technique to a mixture of iron oxide nanocrystals of magnetite and maghemite. The software that goes with this hardware can also be used off line for the creation of nanocrystal orientation and phase maps from HRTEM images. This will be demonstrated for clausthalite nanocrystals that were imaged in an objective lens-aberration corrected TEM.

\section{AUTOMATED NANOCRYSTAL PHASE AND ORIENTATION MAPPING FROM PRECESSION ELECTRON DIFFRACTION DATA}

Precession electron diffraction patterns are automatically recorded by an external digital camera while the area of interest is scanned in a step like fashion with a nanometer-sized electron beam. The data collection step is followed by automatic data processing. Figure 1 gives an overview of the system.

The orientation and phase identification is performed by the matching of each experimental diffraction pattern to a large number of templates, which are computer calculated diffraction patterns for all orientations over the stereographic triangle. For example, a $1^{\circ}$ angular resolution of a highly symmetric crystal phase (such as magnetite) requires fewer than 2,000 templates, Fig. 2

Figure 3 shows the matching procedure schematically. A typical rate for the matching of electron diffraction spot patterns of cubic nanocrystals is approximately 100 patterns per second. The degree of matching between the experimental electron diffraction spot patterns (Fig. 3a) and the calculated templates (Fig. 3b) is given by a correlation index $[3,6,7]$.

The correlation index is calculated for every orientation and plotted on a map that represents two standard stereographic triangles for cubic point groups, Fig. 3c. The resulting map reveals the most probable crystal orientation for every experimental diffraction pattern. The highest value of the index corresponds to the appropriate crystal orientation and phase $[3,6,7]$.

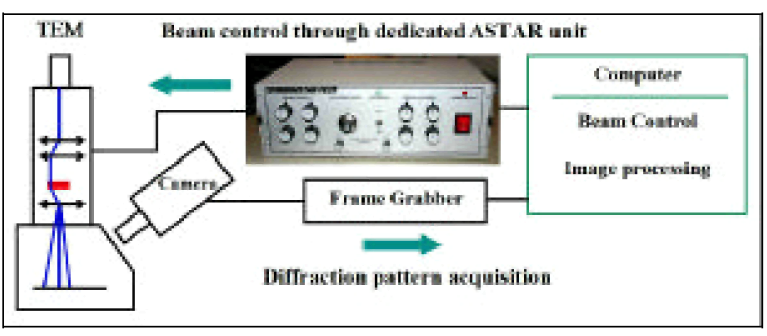

FIGURE 1. The experimental set up of the ASTAR system (from NanoMEGAS) for crystal orientation and phase mapping [3].

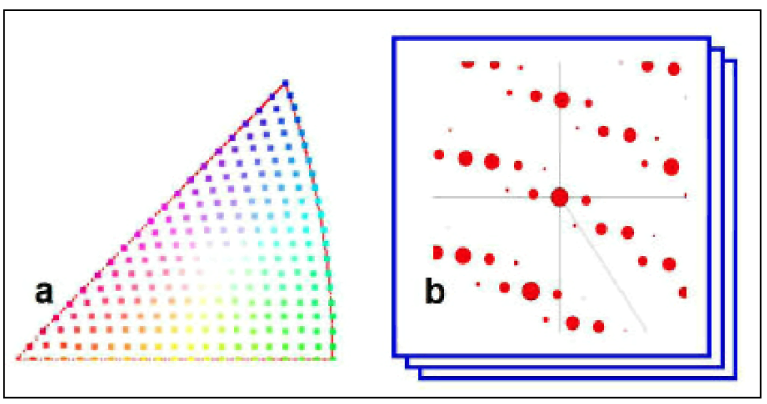

FIGURE 2. (a) The stereographic triangle for cubic crystals with point groups $\mathrm{m} 3 \mathrm{~m}$ and $\overline{4} 3 \mathrm{~m}$; (b) Sketches of templates that are calculated for all orientations over this stereographic triangle.

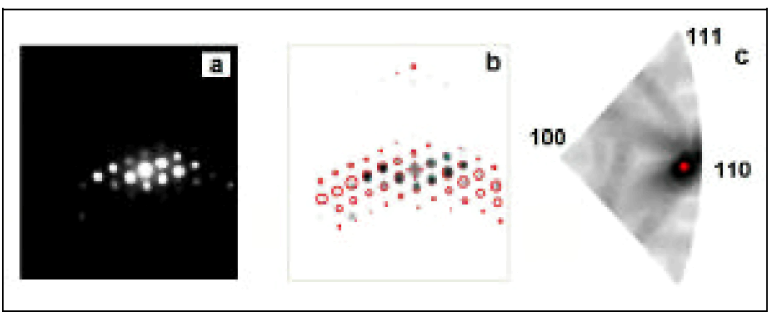

FIGURE 3. Electron diffraction pattern matching: an experimental nano-electron diffraction spot pattern from a crystallite acquired by an external digital camera (a) is compared to each simulated template until the best match is found (b). The correlation index map (c) reflects the degree of matching between experimental patterns and templates. The dot in (c) shows the most probable orientation of the crystallite that led to the electron diffraction spot pattern of (a).

This brief introduction to the automated crystal phase and orientation mapping technique is complemented by a presentation of some results of our study on nanocrystalline iron oxide powders, Fig. 4. Thousands of electron diffraction patterns have been recorded and analyzed automatically in order to generate the crystal orientation and phase maps of the ensembles of iron-oxide nanocrystals in Figs. 5 to 7 .

The spatial resolution of the crystal orientation and phase maps depends on the primary electron beam step size. In our study, this step size was approximately 30 
$\mathrm{nm}$ while the primary electron beam had a diameter of about $20 \mathrm{~nm}$.

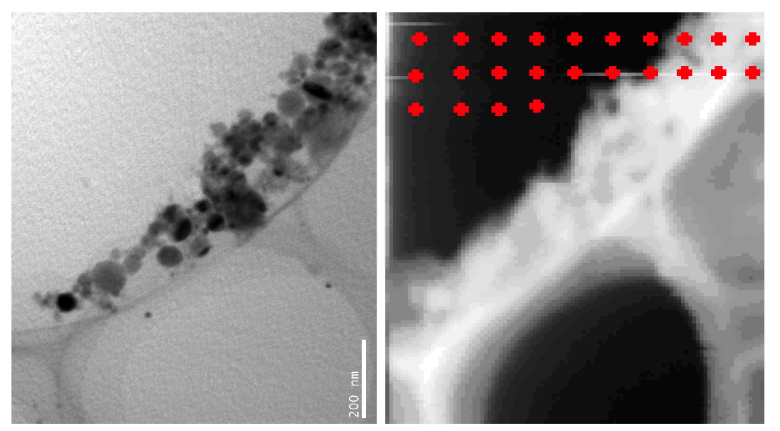

FIGURE 4. Iron oxide nanocrystals in a transmission electron microscope. (left) Standard bright field TEM image. (right) Map of the fluctuations of the primary electron beam intensity in the electron diffraction patterns, analogous to a scanning TEM bright field image. A few positions of the primary electron beam at which electron diffraction patterns were automatically recorded by the external digital camera are illustrated by the added (red) dots.
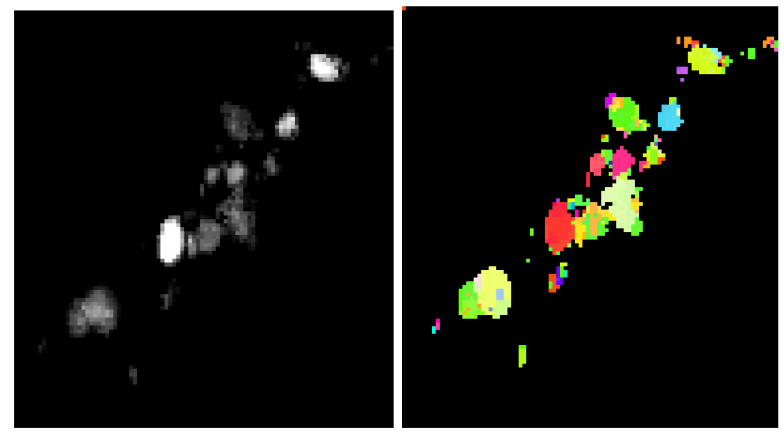

FIGURE 5. Crystal orientation mapping of iron oxide nanocrystals: correlation index (left) and orientation (right) maps, derived from ordinary (not precessed) electron diffraction spot patterns.

In order to demonstrate the effect of a precessing primary electron beam on the reliability of the technique, the analysis was performed twice for the same ensemble of nanocrystals. The same scanning step sizes were used first with an electron beam that was not precessing, Fig. 5, and then with an electron beam that was precessing at approximately $0.3^{\circ}$ around the optical axis of the microscope, Fig. 6.

The comparison of figures 5 and 6 shows clearly that a precessing primary electron beam improves the correlation index and crystal orientation maps significantly. Fig. 7 (left hand side) shows the corresponding crystal phase map for magnetite. A comparison with the correlation index map (right hand side of Fig. 7) shows that the majority of the crystals are of the magnetite phase. The same conclusion was also reached from our earlier studies of HRTEM images and precession electron diffractograms of individual crystallites of this mixture [8-10].
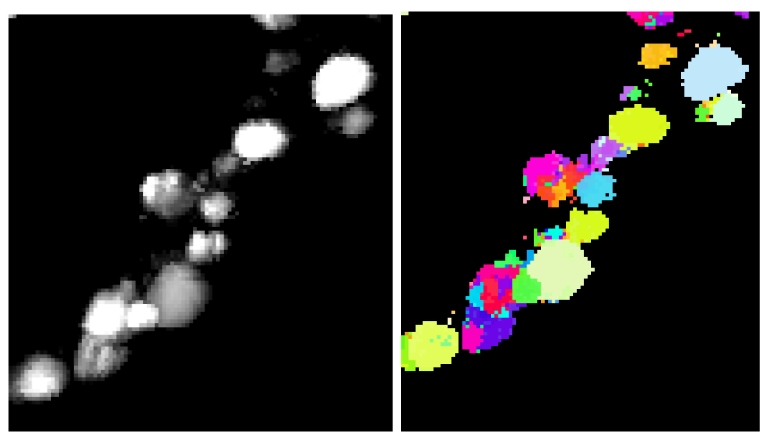

FIGURE 6. Crystal orientation mapping of iron oxide nanocrystals: correlation index (left) and orientation (right) maps, derived from precession electron diffraction spot patterns.
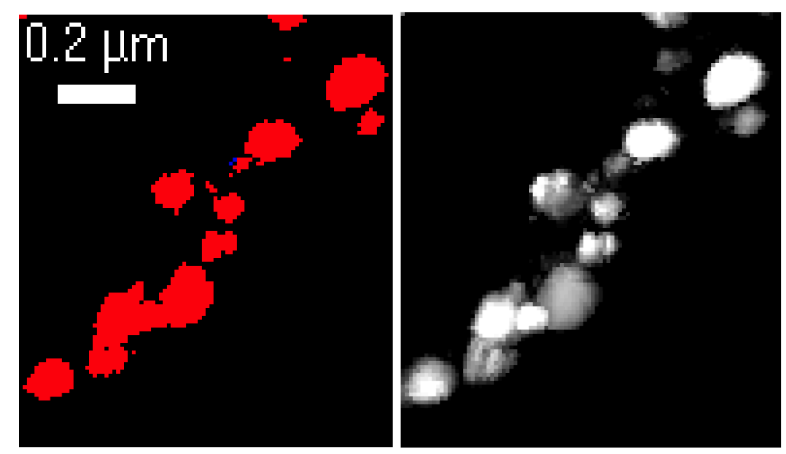

FIGURE 7. Crystal orientation mapping of iron oxide nanocrystals: phase map for magnetite (left) and correlation index (right) map of the whole ensemble of nanocrystals, derived from precession electron diffraction spot patterns.

\section{NANOCRYSTAL PHASE AND ORIENTATION MAPPING FROM HRTEM IMAGES}

An extension of this automated analysis approach to Fourier transforms of parallel-illumination HRTEM images of nanocrystals is illustrated in Fig. 8. The experimental data for this application was recorded on the internal slow-scan CCD camera of an objectivelens aberration-corrected TEM.

The projected reciprocal lattice geometry of a nanocrystal is revealed by the modulus part of the Fourier transform (which is typically displayed in conjunction with HRTEM images and considered to be the "digital diffractogram" of the former). Just as ordinary diffraction patterns, this part of the Fourier transform of HRTEM images can be utilized for the first step of advanced structural fingerprinting [5,8-13], i.e. the determination of the projected reciprocal lattice geometry.

While nanocrystals with relatively large unit cells, i.e. magnetite and maghemite $(\mathrm{a} \sim 0.84 \mathrm{~nm})$, can be structurally fingerprinted in the HRTEM imaging mode with modern analytical TEMs or older "dedicated 
HRTEMs" (with typical point-to-point resolutions of 0.24 to $0.19 \mathrm{~nm}$, e.g. refs. [8-10]), many other inorganic nanocrystals require the utilization of aberration-corrected microscopy for the recording of the data.

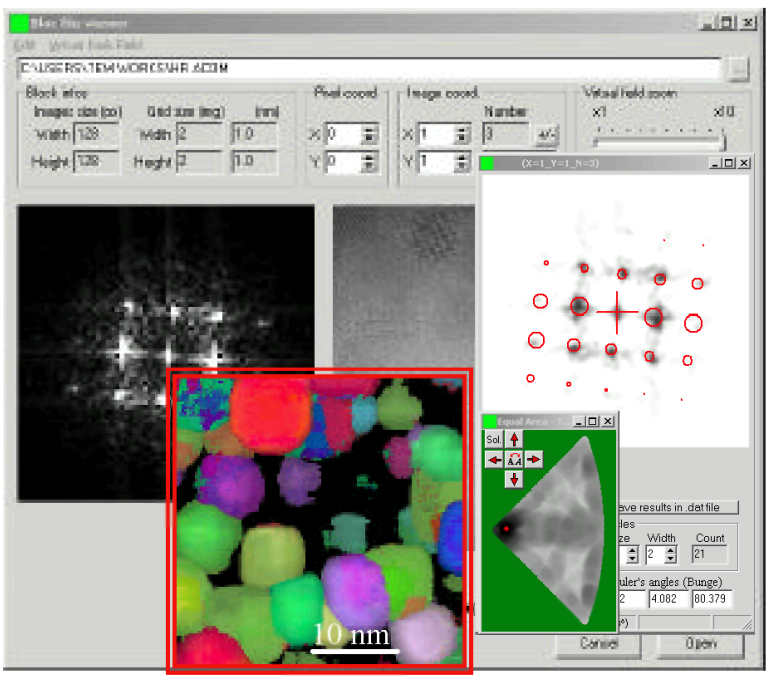

FIGURE 8. Processing of HRTEM images of $\mathrm{PbSe}(\mathrm{a}=0.61$ $\mathrm{nm})$ nanocrystals with software that complements the ASTAR* system. Background window: Overview screenshot of HRTEM image for selection of nanocrystals for calculation of fast Fourier transforms (FFTs). Narrow front window (on the right hand side): Simulated template close to (001) that matches the displayed FFT best (upper part of this window); overlaid smaller front window: Location of that particular template in the stereographic triangle for point groups $432, m \overline{3}$, and 23 . Final doubly framed overlay map: Color-coded crystallite orientation map of PbSe nanocrystals.

Table I gives the crystallographic indices of lattice fringes and zone axes that can (at least in principle) be derived from HRTEM images as a function of the point-to-point resolution of a TEM. A very densely packed, hypothetical, cubic $\mathrm{AB}$-compound with halite structure with a small lattice constant was chosen as example. While the top two rows of this table refer to dedicated HRTEMs, the bottom two rows refer to objective-lens aberration-corrected TEMs.

Besides its obvious utility for a wide range of materials science applications, automated crystallite orientation and phase mapping in a TEM from either single crystal PED patterns or HRTEM images complements both advanced structural fingerprinting from individual nanocrystals [5,8-13] and structural electron crystallography [14]. This is because the crystallite orientation maps may be used for the selection of individual nanocrystals that are oriented close to major zone axes. (For the background and procedures of advanced structural fingerprinting from HRTEM images and PED patterns that include information on plane and $2 \mathrm{D}$ point symmetry groups, structure factor amplitudes, and structure factor phases (in case of HRTEM images), in addition to information on the projected reciprocal lattice geometry, see refs. $[5,8-13])$

Table I: Relationship between point-to-point resolution of a TEM and the visibility of lattice fringes and zone axes within one stereographic triangle [001]-[011]-[111] for a hypothetical cubic AB-compound with $0.425 \mathrm{~nm}$ lattice constant and space group $F m \overline{3} m$, (i.e. the halite structural prototype to which $\mathrm{PbSe}$ belongs).

\begin{tabular}{|c|c|c|}
\hline $\begin{array}{l}\text { Point-to } \\
\text { point } \\
\text { resolution } \\
\text { [nm] }\end{array}$ & $\begin{array}{l}\text { Number and type of } \\
\text { visible lattice } \\
\text { fringes (net-plane } \\
\text { families) }\end{array}$ & $\begin{array}{l}\text { Number and type of } \\
\text { visible zone axes } \\
\text { (lattice fringe } \\
\text { crossings) }\end{array}$ \\
\hline 0.2 & 2, i.e. $\{111\},\{200\}$ & 2, i.e. [001], [011] \\
\hline 0.15 & $\begin{array}{l}3, \text { i.e. }\{111\},\{200\} \\
\{220\}\end{array}$ & $\begin{array}{l}2^{2} \text {, i.e. [001], [011], } \\
{[111],[112]}\end{array}$ \\
\hline 0.1 & $\begin{array}{l}\text { 4, i.e. }\{111\},\{200\} \\
\{220\},\{311\}\end{array}$ & $\begin{array}{l}2^{3}, \text { i.e. [001], [011], } \\
{[111],[112],[013]} \\
{[114],[125],[233]}\end{array}$ \\
\hline$=0.05$ & $\begin{array}{l}=18, \text { i.e. }\{111\}, \\
\{200\},\{220\},\{311\}, \\
\{331\},\{420\},\{422\}, \\
\{511\},\{531\},\{442\}, \\
\{620\},\{622\},\{551\}, \\
\{711\},\{640\},\{642\}, \\
\{731\},\{820\}\end{array}$ & $\begin{array}{l}>2^{5}, \text { e.g. [001], } \\
\text { [011], [111], [012], } \\
\text { [112], [013], [122], } \\
\text { [113], [114], [123], } \\
\text { [015], [133], [125], } \\
\text { [233], [116], [134], } \\
{[035], \ldots}\end{array}$ \\
\hline
\end{tabular}

\section{CRYSTALLOGRAPHIC DATABASES}

Crystal phase identification as outlined above may rely on data from the Powder Diffraction File [15], the Crystal Data database of the National Institute of Standards and Technology (NIST) [16], the Inorganic Crystal Structure Database [17], the NIST Standard Reference Data Bases 83 and 84 [18], and the Pearson's Crystal Data [19] database.

As an alternative to these commercial databases, one may use comprehensive open-access databases. More than 100,000 comprehensive datasets are currently available for inorganic crystals at the combined web sites of the Crystallography Open Database (COD) $[20,21]$, the COD's mainly inorganic and educational subset [22], the American Mineralogist Crystal Structure Database [23], and the Linus Pauling File [24]. Note that the COD has currently more than 80,500 comprehensive entries and is rapidly growing [21].

\section{SUMMARY AND CONCLUSIONS}

The automated crystal phase and orientation mapping technique enables fast and reliable analyses of ensembles of nanocrystals. Since it is well known that the precession of the primary electron beam increases 
the number of diffraction spots in electron diffraction patterns while dynamical electron diffraction effects are suppressed for crystal thicknesses of less than approximately $50 \mathrm{~nm}$, it is no surprise that automated crystal phase and orientation mapping benefits from precession electron diffraction.

Our study shows also that this transmission electron microscopy based electron diffraction spot pattern technique is capable of solving problems for which there are no solutions by electron backscattering techniques of scanning electron microscopy.

\section{ACKNOWLEDGMENTS}

This research was supported by grants from the Oregon Nanoscience and Microtechnologies Institute. Dr. Klaus H. Pecher from the Pacific Northwest National Laboratory is thanked for the nanocrystalline iron-oxide sample. Prof. Marie Cheynet of the Institut National Polytechnique de Grenoble is thanked for the HRTEM image in the background of Fig. 8 (which was taken with the aberration-corrected FEI Titan 80-300 microscope at the Canadian Centre for Electron Microscopy at McMaster University in Hamilton /Ontario) from $\mathrm{PbSe}$ nanocrystals that were produced by Dr. Odile Robbe of the Université de Lille.

\section{REFERENCES}

1. D. Dingley, J. Microscopy 213 (3), 214-224 (2004)

2. C. Rao, P. J. Thomas, and G. Kulkarni, Nanocrystals: Synthesis, Properties and Applications, Springer Series in Materials Science, Vol. 95, 2007

3. E. Rauch, M. Véron, J. Portillo, D. Bultreys, Y. Maniette, and S. Nicolopoulos, Microscopy and Analysis, Issue 93, November 2008, S5-S8

4. R. Vincent and P. Midgley, Ultramicroscopy 53, 271282 (1994)

5. P. Moeck and S. Rouvimov, in: Drug Delivery Nanoparticles: Formulation and Characterization, Y. Pathak and D. Thassu (editors.), Informa, 2009 (Drugs and the Pharmaceutical Sciences, Vol. 191), New York, pp. $270-313$, in press

6. E. Rauch and L. Dupuy, Archives of Metallurgy and Materials 50 (1), 87-99 (2005)

7. E. Rauch and M. Veron, Mat. -wiss. u. Werkstofftech. 36 (10), 552-556 (2005)

8. P. Moeck, R. Bjorge, E. Mandell, and P. Fraundorf, NSTI-Nanotech 2007, Vol. 4, 93-96 (2007), (ISBN 1420063766, www.nsti.org)

9. R. Bjorge, MSc thesis, Portland State University, May 9, 2007; Journal of Dissertation Vol. 1 (2007), $\mathrm{http}: / /$ www.scientificjournals.org/journals $2007 / j_{\text {jof_disse }}$ rtation.htm

10. P. Moeck and R. Bjorge, in: Quantitative Electron Microscopy for Materials Science, Mater. Res. Soc. Symp.
Proc. Vol. 1026E paper 1026-C17-10 (2007), editors: E. Snoeck, R. Dunin-Borkowski, J. Verbeeck, and U. Dahmen

11. P. Moeck and P. Fraundorf, Zeitschrift für

Kristallographie 222, 634-645 (2007), expanded version at arXiv:0706.2021

12. P. Moeck, S. Rouvimov, S. Nicolopoulos, and P. Oleynikov, NSTI-Nanotech 2008, Vol. 1, 912-915, ISBN 978-1-4200-8503-7, www.nsti.org, see also arXiv:0804.0063

13. P. Moeck, S. Rouvimov, E. Rauch and S Nicolopoulos, Mater. Res. Soc. Proc. Vol. 1184, paper 1184-GG03-07 (2009), editors: P. Moeck, S. Hovmoeller, S. Nicolopoulos, S. Rouvimov, V. Petkov, M. Gateshki, and P. Fraundorf

14. X. D. Zou and S. Hovmöller, Acta Cryst. A 64, 149160 (2008); http://journals.iucr.org/a/issues/2008/01/00/ issconts.html

15. http://www.icdd.com

16. http://www.nist.gov/srd/nist3.htm

17. http://www.fiz-karlsruhe.de/icsd.html

18. http://www.nist.gov/srd/nist83.htm and http://www.nist.gov/srd/nist84.htm

19. http://www.crystalimpact.com/ped/Default.htm

20. http://www.crystallography.net mirrored at:

http://cod.ibt.lt (in Lithuania), http://cod.ensicaen.fr/ (in France), http://nanocrystallography.org and http://nanocrystallography.net (both in Oregon, USA), also accessible under a different search surface at: http://fireball.phys.wvu.edu/cod/ (in West Virginia, USA) 21. S. Gražulis, D. Chateigner, R. T. Downs, A. F. T. Yokochi, M. Quirós, L. Lutterotti, E. Manakova, J. Butkus, P. Moeck, and A. Le Bail, J. Appl. Cryst. 42 (part 4), August 2009, http://journals.iucr.org/j/issues/2009/04/ 00/kk5039/kk5039.pdf, in press

22. http://nanocrystallography.research.pdx.edu/CIFsearchable/cod.php, data on some 20,000 crystals 23. http://ruff.geo.arizona.edu/AMS/amesd.php, data on some 10,000 minerals

24. http://crystdb.nims.go.jp, data on some 30,000 metals and alloys

* Peter Moeck's "Laboratory for Structural Fingerprinting and Electron Crystallography" at Portland State University serves as the first demonstration site of the NanoMEGAS company in the Americas. A first generation (analog) "Spinning Star" precession electron diffraction device is currently interfaced there to an analytical field-emission TEM and can be demonstrated on request (to pmoeck@pdx.edu or tel.: 503725 4227). By the end of 2009, this laboratory will also possess a second generation (digital) "DigiStar" precession electron diffraction device and the ASTAR system so that demonstrations of this so far quite unique equipment will also become possible. 